\title{
GW23-e0389 EFFECTS OF RENAL SYMPATHETIC DENERVATION ON RATS WITH HEART FAILURE AFTER MYOCARDIAL INFARCTION
}

doi:10.1136/heartjnl-2012-302920b.34

\author{
${ }^{1}$ Jialu Hu, ${ }^{2}$ Meng Ji, ${ }^{2}$ Asya, ${ }^{2}$ Yuemei Hou, ${ }^{1}$ Yan Yan. ${ }^{1}$ Department of Cardiology, \\ Zhongshan Hospital, Fudan University, Shanghai 200032; ${ }^{2}$ First Affiliated Hospital of \\ Xinjiang Medical University 830001
}

Objectives In recent years, some clinical trials have demonstrated that Renal denervation (RD) of radio frequency ablation has improved sodium and water retention and the chronic over-activation of the sympathetic nerve system, making the treatment of refractory hypertension achieve a breakthrough. In this study we want to investigate the therapeutic effects of $\mathrm{RD}$ on rats with heart failure after myocardial infarction, as well as the optimal time for performing RD and the sustainability of these effects.

Methods 120 SPF male Wistar rats (SPF level, 280-330 g) were randomly assigned to six groups, that is, rats with myocardial infarction (MI), rats with $\mathrm{RD}$, rats with $\mathrm{RD} 3+\mathrm{MI}$ (myocardial infarction 3 days after renal denervation), rats with $\mathrm{MI} 1+\mathrm{RD}$ (renal denervation 1 day after $\mathrm{MI}$ ), rats with MI7+RD (renal denervation 7 days after MI), rats with MI4W+RD (renal denervation 4 weeks after $\mathrm{MI}$ ) and normal control rats. 8 weeks after MI, the body eight, the left ventricular weight, the urine volume, the urinary sodium, the left ventricular function, the BNP content in serum, the NE content of cardiac tissue were measured.

\section{Results}

1. Ligation of the left anterior descending coronary artery in rats induced MI, and the myocardial infarct size was more than $20 \%$. The left ventricular function of MI group significantly declined. Eight weeks after MI, EF and FS of MI group were significantly reduced from $87 \pm 4.2 \%$ and $42 \pm 5.6 \%$ to $37 \pm 6 \%$ and $16 \pm 3 \%(p<0.05)$. The urine output and urine sodium excretion were markedly reduced from $23.83 \pm 1.9 \mathrm{ml}$ and 0.259 $\pm 0.061 \mathrm{mmol}$ to $14.13 \pm 3.8 \mathrm{ml}$ and $0.138 \pm 0.019 \mathrm{mmol}$ $(p<0.05)$.

2. Surgically stripping the adventitia of renal arteries and veins, approximately $3 \mathrm{~mm}$ from the abdominal aorta can achieve denervation (By observing the blood pressure, heart rate and renal colour changes.).

3. In rats with $\mathrm{RD} 3+\mathrm{MI}, \mathrm{MI} 1+\mathrm{RD}, \mathrm{MI} 7+\mathrm{RD}$ and $M I 4 \mathrm{~W}+\mathrm{RD}$ the left ventricular function significantly improved compared with that in rats with MI. Additionally, the serum BNP content decreased significantly $(p<0.05)$, the urine output increased $(p<0.05)$, and the urinary sodium excretion also increased but without any significant difference ( $p>0.05)$.

Conclusions $\mathrm{RD}$ has preventive and therapeutic effects on heart failure after MI through improving left ventricular remodelling, 


\section{ABSTRACTS}

cardiac function and water excretion, and the effects can sustain at least 8 weeks, but there were no significant differences for different time points of denervation. Cardiac function, urine volume and urine sodium in normal rats were not affected by $\mathrm{RD}(\mathrm{p}>0.05)$. $\mathrm{RD}$ was subjected to an in-depth study on the mechanism of improving heart failure after MI. 